\title{
金属-配体协同作用下钉催化剂用于草酸酯加氢制乙二醇的研究进展
}

\author{
方霄龙 $*, a, b$ \\ 段 宁 ${ }^{a}$ 章 敏 ${ }^{a}$ \\ 李斌*,b \\ ( ${ }^{a}$ 安徽建筑大学材料与化学工程学院 功能分子设计与界面过程重点实验室 合肥 230601) \\ ( ${ }^{b}$ 厦门大学化学化工学院 固体表面物理化学国家重点实验室 醇梄酯清洁生产国家工程实验室 厦门 361005)
}

\begin{abstract}
摘要 乙二醇是一种重要的基础化工原料, 被广泛用于生产聚酷等工业化学品. 催化草酸酯加氢制乙二醇是 “煤制乙 二醇” 工业路线的关键步骤. 研究开发可高效催化草酸酯加氢的催化剂是化学家关注的研究热点. 调研并总结了近 10 年来具有金属一配体协同作用的钓催化剂用于草酸酯均相催化加氢的研究进展, 围绕催化剂结构与性质的关联, 探讨 催化加氢反应机理, 为进一步开发性能优异的新型催化剂提供参考.
\end{abstract}

关键词＼cjkstart草酸酯加氢; 乙二醇; 金属-配体协同作用; 钉催化剂

\section{Advances for Ruthenium Catalysts with Metal-Ligand Cooperation for Hydrogenation of Oxalates into Ethylene Glycol}

\author{
Fang, Xiaolong*,a,b \\ Duan, Ning ${ }^{a}$ \\ Zhang, $\operatorname{Min}^{a}$ \\ Li, $\operatorname{Bin}^{*, b}$ \\ ( ${ }^{a}$ Key Laboratory of Functional Molecule Design and Interface Process, College of Materials and Chemical Engineering, \\ Anhui Jianzhu University, Hefei 230601) \\ ( $b$ State Key Laboratory of Physical Chemistry of Solid Surfaces, National Engineering Laboratory for Green Chemical Pro- \\ ductions of Alcohols-Ethers-Esters, College of Chemistry and Chemical Engineering, Xiamen University, Xiamen 361005)
}

\begin{abstract}
Ethylene glycol (EG) is an important fundamental chemical material, which has been widely used in the production of industrial chemicals such as polyester. Catalytic hydrogenation of oxalate into EG is a key step in the "coal-to-EG" industrial route. Design of efficient catalysts for catalyzing the hydrogenation of oxalate esters is a research hotspot. The research progress of ruthenium catalysts with metal-ligand cooperation in the homogeneous catalytic hydrogenation of oxalate esters in the past decade is reviewed. Based on the relationship between the catalyst structures and properties, the catalytic hydrogenation mechanism is discussed, which provides a reference for the further design and development of new catalysts with excellent performance.
\end{abstract}

Keywords hydrogenation of oxalate; ethylene glycol; metal-ligand cooperation; ruthenium catalyst

聚酯切片是连接石化产品和纺织、建筑以及包装等 多个行业的重要中间产品. 我国作为世界上最大的聚酯 生产国和消费国, 聚酯工业的发展对我国国民经济的发 展有重要意义. 聚对苯二甲酸乙二醇酯(PET)作为最重 要的聚酯品种之一，拥有优良的机械性能，同时具有耐 磨及尺寸稳定性好等优点 ${ }^{[1]}$. 乙二醇(EG) 是 PET 的主要 生产原料之一. 近年来, 虽然我国的 $\mathrm{EG}$ 产量取得了显 著的增长, 但仍不能满足市场的强烈需求, 约 $70 \%$ 的 $\mathrm{EG}$ 依赖于进口 ${ }^{[2]}$. 因此, 大力发展 EG 的生产进而缓解 供需矛盾是目前广泛研究的领域之一.
目前，乙二醇的制备方法有乙烯直接水合法、二氯 乙烷水解法、碳酸乙烯酯法、环氧乙烷水合法和草酸酯 法等(表 1). 乙烯直接水合法等均属于传统的石油化工 路线, 原料乙烯依赖于石油的裂解(表 1, Entries 1 4). 草酸酯法由合成气 $\left(\mathrm{CO}+2 \mathrm{H}_{2}\right)$ 出发经草酸酯加氢制备 EG，属于现代煤化工路线(表 1, Entry 5). 由于乙烯直接 水合法、二氯乙烷水解法等存在设备腐蚀严重和成本高 等不足，到 20 世纪 70 年代就已经基本被淘汰，当前工 业上应用较多的是环氧乙烷水合法和草酸酯法 ${ }^{[3]}$. 考虑 到我国 “富煤贫油” 的能源结构, “煤制乙二醇” 工艺

\footnotetext{
* Corresponding authors. E-mail: xlfang@stu.xmu.edu.cn; binlee@stu.xmu.edu.cn Received April 12, 2020; revised May 9, 2020; published online May 27, 2020. Project supported by the National Natural Science Foundation of China (No. 21802010) and the Natural Science Foundation of Anhui Province (No. 1808085QB48).

国家自然科学基金(No. 21802010)和安徽省自然科学基金(No. 1808085QB48)资助项目.
} 
表 1 乙二醇的工业制备方法及其优缺点

Table 1 Advantages and disadvantages of industrial preparation of ethylene glycol

\begin{tabular}{|c|c|c|c|c|c|}
\hline Entry & 制备方法 & 原料 & 制备过程 & 优势 & 不足 \\
\hline 1 & 乙烯直接水合法 & 乙烯 & $\mathrm{C}_{2} \mathrm{H}_{4}+1 / 2 \mathrm{O}_{2}+\mathrm{H}_{2} \mathrm{O} \rightarrow \mathrm{HOCH}_{2} \mathrm{CH}_{2} \mathrm{OH}$ & 原料成本较低 & $\begin{array}{l}\text { 设备腐蚀严重、 } \\
\text { 分离精馏成本高 }\end{array}$ \\
\hline 2 & 二氯乙烷水解法 & 乙烯 & $\begin{array}{l}\mathrm{C}_{2} \mathrm{H}_{4}+\mathrm{Cl}_{2} \rightarrow \mathrm{ClCH}_{2} \mathrm{CH}_{2} \mathrm{Cl} \\
\mathrm{ClCH}_{2} \mathrm{CH}_{2} \mathrm{Cl}+2 \mathrm{H}_{2} \mathrm{O} \rightarrow \mathrm{HOCH}_{2} \mathrm{CH}_{2} \mathrm{OH}+2 \mathrm{HCl}\end{array}$ & - & $\begin{array}{c}\text { 设备腐蚀严重、 } \\
\text { 总成本高 }\end{array}$ \\
\hline 3 & 碳酸乙烯酯法 & 乙烯 & $\begin{array}{l}\mathrm{C}_{2} \mathrm{H}_{4}+1 / 2 \mathrm{O}_{2} \rightarrow \mathrm{C}_{2} \mathrm{H}_{4} \mathrm{O} \\
\mathrm{C}_{2} \mathrm{H}_{4} \mathrm{O}+\mathrm{CO}_{2} \rightarrow\left(\mathrm{CH}_{2} \mathrm{O}\right)_{2} \mathrm{CO} \\
\left(\mathrm{CH}_{2} \mathrm{O}\right)_{2} \mathrm{CO}+\mathrm{H}_{2} \mathrm{O} \rightarrow \mathrm{HOCH}_{2} \mathrm{CH}_{2} \mathrm{OH}+\mathrm{CO}_{2}\end{array}$ & $\begin{array}{l}\text { 反应条件温和、 } \\
\text { 能耗低 }\end{array}$ & 设备总投资大 \\
\hline 4 & 环氧乙烷水合法 & 乙烯 & $\begin{array}{l}\mathrm{C}_{2} \mathrm{H}_{4}+1 / 2 \mathrm{O}_{2} \rightarrow \mathrm{C}_{2} \mathrm{H}_{4} \mathrm{O} \\
\mathrm{C}_{2} \mathrm{H}_{4} \mathrm{O}+\mathrm{H}_{2} \mathrm{O} \rightarrow \mathrm{HOCH}_{2} \mathrm{CH}_{2} \mathrm{OH}\end{array}$ & 乙二醇收率高 & $\begin{array}{l}\text { 催化剂选择性、 } \\
\text { 稳定性有待提高 }\end{array}$ \\
\hline 5 & 草酸酯法 & 合成气 & $\begin{array}{l}2 \mathrm{CO}+1 / 2 \mathrm{O}_{2}+2 \mathrm{ROH} \rightarrow \mathrm{ROOCCOOR}+\mathrm{H}_{2} \mathrm{O} \\
\mathrm{ROOCCOOR}+4 \mathrm{H}_{2} \rightarrow \mathrm{HOCH}_{2} \mathrm{CH}_{2} \mathrm{OH}+2 \mathrm{ROH}\end{array}$ & $\begin{array}{l}\text { 反应条件温和、 } \\
\text { 工艺要求低 }\end{array}$ & $\begin{array}{l}\text { 催化剂选择性、 } \\
\text { 稳定性有待提高 }\end{array}$ \\
\hline
\end{tabular}

路线的发展应用有望协助打破我国大宗工业用乙二醇 依赖于进口的严峻局面. 在草酸酯法中, 草酸酯的制备 技术已经成熟，因而高效催化草酸酯加氢制 EG 是这一 路线的研究重点和难点 ${ }^{[1,4]}$.

催化草酸酯加氢制 $\mathrm{EG}$ 是一连续的反应过程, 首先 一个酯基加氢生成中间产物乙醇酸酯, 乙醇酸酯进一步 加氢生成 EG (Scheme 1). 此外, 还可能发生 EG 过渡加 氢生成乙醇以及乙醇与 $\mathrm{EG}$ 分子间脱水生成 1,2-丁二醇 等副反应. 由于草酸酯相邻酯基间的活化作用, 催化草 酸酯部分加氢制乙醇酸酯反应难度低于催化乙醇酸酯 加氢制 $\mathrm{EG}^{[5]}$. 催化剂在这一加氢反应过程中扮演着至 关重要的作用. 目前, 可用于草酸酯加氢的催化剂主要 有非均相负载型金属催化剂和均相金属配合物催化剂 两类. 相比于非均相负载型金属催化剂存在反应条件苛 刻(180 $\left.{ }^{\circ} \mathrm{C} 、 20 \mathrm{MPa} \mathrm{H}_{2}\right)$ 、目标产物选择性低、催化加氢 反应机理不明确等问题 ${ }^{[6]}$, 均相金属配合物催化剂可以 在温和条件下高选择性催化底物加氢, 有一定的优势. 同时均相催化剂明确的结构以及对反应过程的原位跟 踪分析, 对于催化反应机理的研究具有重要意义. 开展 草酸酯均相催化加氢的研究, 一方面可以加深对这一反 应过程的理解, 另一方面可以对进一步开发高效的负载 型金属催化剂起指导借鉴的作用.

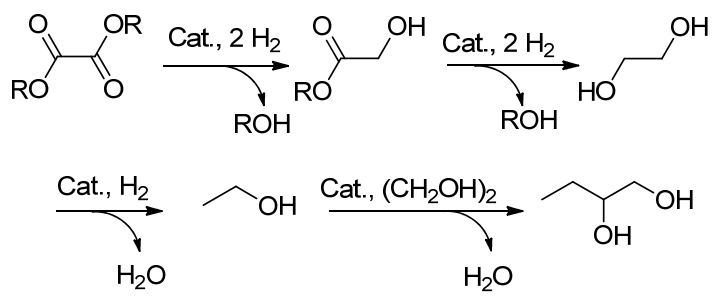

图式 1 催化草酸酯加氢反应过程

Scheme 1 Reaction process for the catalytic hydrogenation of oxalates
1980 年, Grey 和 Pez 等 ${ }^{[5,7]}$ 首次报道了草酸二甲酯 $(\mathrm{DMO})$ 的均相催化加氢研究. 发现钉氢负离子 $\left[\left(\mathrm{Ph}_{3} \mathrm{P}\right)_{2}-\right.$ $\left.\mathrm{Ph}_{2} \mathrm{P}\left(\mathrm{C}_{6} \mathrm{H}_{4}\right) \mathrm{RuH}_{2}\right]^{-}$和 $\left[\left(\mathrm{Ph}_{3} \mathrm{P}\right)_{3}\left(\mathrm{Ph}_{2} \mathrm{P}\right) \mathrm{Ru}_{2} \mathrm{H}_{4}\right]^{2-}$ 可以催化 $\mathrm{DMO}$ 加氢制乙醇酸甲酯 $(\mathrm{MG})$; 其中 $\left[\left(\mathrm{Ph}_{3} \mathrm{P}\right)_{3}\left(\mathrm{Ph}_{2} \mathrm{P}\right)-\right.$ $\left.\mathrm{Ru}_{2} \mathrm{H}_{4}\right]^{2-}$ 的效果较好, 在 $90{ }^{\circ} \mathrm{C}$ 和 $0.62 \mathrm{MPa} \mathrm{H}_{2}$ 条件下反 应 $20 \mathrm{~h}$ 可以完成 $70 \%$ 的 DMO 转化. Matteoli 等 ${ }^{[8]}$ 于 1984 年报道了钉羰基氢化物 $\mathrm{H}_{4} \mathrm{Ru}_{4}(\mathrm{CO})_{8}\left(\mathrm{PBu}_{3}\right)_{4}$ 和 $\mathrm{H}_{4} \mathrm{Ru}_{4}$ $(\mathrm{CO})_{8}\left(\mathrm{PPh}_{3}\right)_{4}$ 催化 DMO 加氢的研究. 活性测试结果表明 在 $180{ }^{\circ} \mathrm{C}$ 和 $13.17 \mathrm{MPa} \mathrm{H}_{2}$ 等反应条件下, $\mathrm{H}_{4} \mathrm{Ru}_{4}(\mathrm{CO})_{8}-$ $\left(\mathrm{PBu}_{3}\right)_{4}$ 可以催化 $\mathrm{DMO}$ 加氢制 $\mathrm{MG}(144 \mathrm{~h}$, 产率 51.1\%), 而 $\mathrm{H}_{4} \mathrm{Ru}_{4}(\mathrm{CO})_{8}\left(\mathrm{PPh}_{3}\right)_{4}$ 没有催化活性. 这是首例关于配体 可以影响钉催化剂在草酸酯加氢反应中催化性能的报 道. 作者研究发现催化剂在反应过程中生成新的钓金属 活性物种 $\mathrm{H}_{4} \mathrm{Ru}_{4}(\mathrm{CO})_{9}\left(\mathrm{PBu}_{3}\right)_{3}$ 和 $\mathrm{H}_{4} \mathrm{Ru}_{4}(\mathrm{CO})_{10}\left(\mathrm{PBu}_{3}\right)_{2}$, 但 在文中没有对膦配体的影响机制展开深入的研究和讨 论. 1985 年, 作者进一步将钉羰基化合物 $\mathrm{Ru}(\mathrm{CO})_{2}-$ $(\mathrm{OAc})_{2}\left(\mathrm{PBu}_{3}\right)_{2}$ 应用于该加氢反应研究中 ${ }^{[9]}$. 在 $180{ }^{\circ} \mathrm{C} 、$ $20.0 \mathrm{MPa} \mathrm{H}_{2}$ 等较为苛刻的条件下反应 $144 \mathrm{~h}$ 取得了 $18 \%$ $\mathrm{MG}$ 和 $82 \% \mathrm{EG}$ 收率的催化效果.

1997 年, Elsevier 等 ${ }^{[10]}$ 发现原位催化剂体系 $\mathrm{Ru}(\mathrm{acac})_{3} / \mathbf{L}_{\mathbf{1}} / \mathrm{Zn}$ (acac: 乙酰丙酮)的甲醇溶液在 $100{ }^{\circ} \mathrm{C}$ 、 $7.0 \mathrm{MPa} \mathrm{H}_{2}$ 等条件下可以有效催化 DMO 加氢制 EG (16 $\mathrm{h}$ ，产率 $94 \%$ ). 甲醇和金属 $\mathrm{Zn}$ 在反应过程中均起到还原 $\mathrm{Ru}(\mathrm{acac})_{3}$ 生成活性中心的作用 ${ }^{[11]}$. 对比发现这一催化 体系的催化活性有显著的提升，且反应条件较为温和. 作者也考察了不同膦配体与 $\mathrm{Ru}(\mathrm{acac})_{3}$ 所构成催化剂体 系的加氢性能, 结果呈现出 $\mathrm{P}\left(\mathrm{C}_{6} \mathrm{H}_{11}\right)_{3}<\mathrm{Ph}_{2} \mathrm{PC}_{2} \mathrm{H}_{4} \mathrm{PPh}_{2}<$ $\mathrm{PPh}_{3}<\mathrm{PhP}\left(\mathrm{C}_{2} \mathrm{H}_{4} \mathrm{PPh}_{2}\right)_{2} \approx\left[\mathrm{CH}_{2} \mathrm{P}(\mathrm{Ph}) \mathrm{C}_{2} \mathrm{H}_{4} \mathrm{PPh}_{2}\right]_{2} \ll \mathrm{MeC}-$ $\left(\mathrm{CH}_{2} \mathrm{PPh}_{2}\right)_{3}$ 的活性变化趋势. 2006 年, Hanton 等 ${ }^{[12]}$ 进一 步考察了配体 $\mathbf{L}_{2}$ 与 $\mathrm{Ru}(\mathrm{acac})_{3}$ 所构成催化剂体系的反应 性能. 在 $100{ }^{\circ} \mathrm{C} 、 8.0 \mathrm{MPa} \mathrm{H}_{2}$ 等条件下反应 $136 \mathrm{~h}, \mathrm{DMO}$ 仅转化为 $\mathrm{MG}$, 无 $\mathrm{EG}$ 生成. 这些结果表明配体结构对催 
化性能有显著影响.

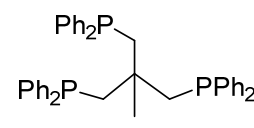

$\mathrm{L}_{1}$<smiles>CCCCCC(C)(C)CC(C)(CBr)CCCC</smiles>

$\mathrm{L}_{2}$
综上所述, 早期用于草酸酯均相加氢的催化剂均为 基于膦配体或硫配体的 $\mathrm{Ru}$ 化合物, 所需反应条件较为 苛刻, 催化活性较低. 1995 年, Noyori 等 ${ }^{[13]}$ 报道指出乙 二胺配体可以显著促进膦- $\mathrm{Ru}(\mathrm{II})$ 催化剂催化醛、酮等羰 基衍生物分子加氢活性. 研究发现反应体系中通过原位 生成的前驱体 trans-[ $\mathrm{RuCl}_{2}$ (phosphine) ${ }_{2}$ (diamine)]形成了 含有钓金属氢 $(\mathrm{RuH})$ 的催化活性物种, 进而在 $\mathrm{RuH}$ 与配 体 NH 的协同作用下通过与湠基发生亲核和亲电作用形 成过渡态(TS) 实现催化活性的提升 ${ }^{[14]}$. 这一机理与传统 的膦- $\mathrm{Ru}$ 催化剂体系有关 $\mathrm{H}_{2}$ 分子活化以及催化加氢反 应过程均在金属中心发生的 “内核加氢反应机理” 截然 不同 ${ }^{[15]}$. 目前, 研究者已经成功制备出一系列具有金属 -配体协同作用的 $\mathrm{Ru}(\mathrm{II})$ 催化剂 ${ }^{[16]} .2007$ 年, Saudan 研究 组 ${ }^{[17]}$ 首次报道将该类型催化剂 $\mathbf{A}$ 和 $\mathbf{B}$ 应用于苯甲酸甲 酯(MB)等多种酯类分子的均相加氢反应研究, 取得了 极大的成功. 近年来, 研究人员也将该类催化剂应用于 草酸酯的均相催化加氢, 在较为温和条件下取得了优异 的催化反应结果, 相比于传统的膦-Ru 催化剂体系有显 著的提升. 本文将对典型的应用于草酸酯均相加氢且具 有金属一配体协同作用的 $\mathrm{Ru}(\mathrm{II})$ 催化剂进行简要论述, 重点探讨催化剂结构与性质的关联以及催化加氢反应 机理.

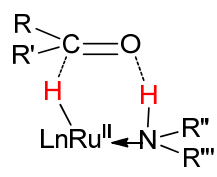

TS

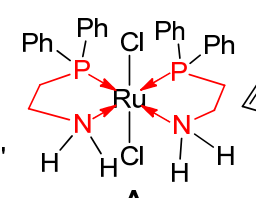

A

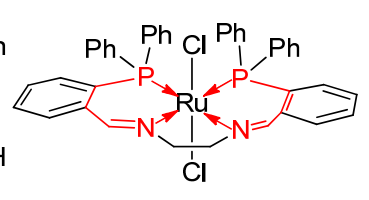

B

\section{1 具有金属-配体协同作用 $\mathrm{Ru}(\mathrm{II})$ 催化剂用于 草酸酯加氢的研究进展}

2011 年以来, Takasago 公司首创的鳌合型 Macho 钉 化合物(Cat. 1, 表 2)已经被成功应用于乳酸甲酯和碳酸 乙烯酯等多种底物分子的加氢反应研究中 ${ }^{[18]}$. Ding 等 ${ }^{[186]}$ 研究提出 $\mathrm{RuH}$ 与配体 $\mathrm{NH}$ 的协同作用是该化合物 取得优异加氢活性的关键. 2013 年, Beller 研究组 ${ }^{[19]}$ 进一 步考察了该类化合物催化草酸二乙酯(DEO)加氢的反应 性能, 结果如表 2 所示. 在 $\mathrm{NaOEt} /$ 四氢呋喃(THF)溶液 中于 $100{ }^{\circ} \mathrm{C} 、 6.0 \mathrm{MPa} \mathrm{H}_{2}$ 等条件下反应 $20 \mathrm{~h}, 1$ 可以催 化 DEO 转化为 EG (表 2, Entry 1). 这一结果与 Gusev
等 ${ }^{[20]}$ 报道的结构相似的 $\mathrm{Os}$ 化合物 $\left(1-\mathrm{CH}_{2} \mathrm{NHCH}_{2}\right.$ $\left.\mathrm{CH}_{2} \mathrm{P}^{i} \mathrm{Pr}_{2} \mathrm{C}_{5} \mathrm{H}_{4} \mathrm{~N}\right) \mathrm{OsHCl}(\mathrm{CO})$ 不能催化草酸酯加氢的研究 结果形成鲜明的对比. $\mathbf{1}$ 的 $\mathrm{NaBH}_{4}$ 还原产物 $\mathbf{2}$ 在相同条 件下催化活性略优于 1, EG 收率达 96\%(表 2, Entry 2). 将 1 结构中 $\mathrm{Ph}_{2} \mathrm{P}$ 替换为 ${ }^{i} \mathrm{Pr}_{2} \mathrm{P}$ 所得化合物 3 以及相应的 氢化物 4 活性均较低, 仅可以催化 $\mathrm{DEO}$ 转化为乙醇酸乙 酯(表 2, Entries 3,4). 对比化合物 $\mathbf{1}$ (或 2)和 3(或 4)反应 结果可以看出催化剂结构对其性能有显著影响.

表 2 不同催化剂体系催化 DEO 加氢 ${ }^{a}$

Table 2 Catalytic hydrogenation of DEO with different catalyst systems

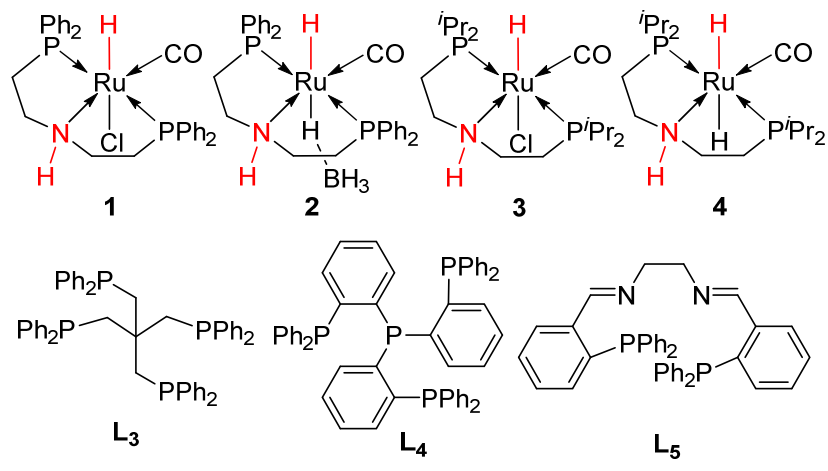

\begin{tabular}{ccccc}
\hline Entry & Cat. & Conv./\% & $\begin{array}{c}\text { Yield/\% of ethyl } \\
\text { glycolate }\end{array}$ & Yield/\% of EG \\
\hline 1 & $\mathbf{1}$ & 100 & 0 & 92 \\
2 & $\mathbf{2}$ & 100 & 0 & 96 \\
3 & $\mathbf{3}$ & 91 & 89 & 0 \\
4 & $\mathbf{4}$ & 100 & 96 & 0 \\
5 & $\mathbf{L}_{\mathbf{1}} / \mathrm{Ru}(\mathrm{acac})_{3}$ & 0 & 0 & 0 \\
6 & $\mathbf{L}_{\mathbf{3}} / \mathrm{Ru}(\mathrm{acac})_{3}$ & 0 & 0 & 0 \\
7 & $\mathbf{L}_{\mathbf{4}} / \mathrm{Ru}(\mathrm{acac})_{3}$ & 4 & 0 & 0 \\
8 & $\mathbf{L}_{\mathbf{5}} / \mathrm{Ru}(\mathrm{acac})_{3}$ & 7 & 0 & 0 \\
$9^{b}$ & $\mathbf{L}_{\mathbf{1}} / \mathrm{Ru}(\mathrm{acac})_{3}$ & 100 & 0 & 96 \\
\hline${ }^{a}$ Reaction conditions: $\mathrm{DEO}(3.7 \mathrm{mmol}), \mathrm{Ru}(0.0201 \mathrm{mmol}), \mathrm{NaOEt}(0.2$
\end{tabular}
mmol), THF $(20 \mathrm{~mL}), p\left(\mathrm{H}_{2}\right)=6.0 \mathrm{MPa}, 100{ }^{\circ} \mathrm{C}, 20 \mathrm{~h} ;{ }^{b} \mathrm{MeOH}$ as solvent.

Beller 等 ${ }^{[19]}$ 也考察了配体 $\mathbf{L}_{1} 、 \mathbf{L}_{3} \sim \mathbf{L}_{5}$ 分别与 $\mathrm{Ru}(\mathrm{acac})_{3}$ 所构成催化剂体系在相同条件下催化 DEO 加 氢的反应性能. THF 溶剂中膦配体 $\mathbf{L}_{\mathbf{1}}$ (或 $\mathbf{L}_{\mathbf{3}} 、 \mathbf{L}_{\mathbf{4}}$ )与 $\mathrm{Ru}(\mathrm{acac})_{3}$ 组成的催化剂体系没有催化活性(表 2 , Entries 5 7); 而在甲醇溶剂中, $\mathbf{L}_{1} / \mathrm{Ru}(\mathrm{acac})_{3}$ 催化活性与催化 剂 $\mathbf{1}$ (或 2)相当(表 2, Entry 9). 这一结果与 Elsevier 等 ${ }^{[10]}$ 的研究结果相吻合, 表明甲醇在活化 $\mathbf{L}_{\mathbf{1}} / \mathrm{Ru}(\mathrm{acac})_{3}$ 生成 催化活性物种这一过程中不可或缺. 如前文所述, 配体 $\mathbf{L}_{5}$ 与 $\mathrm{Ru}(\mathrm{II})$ 配位所构成的化合物 $\mathbf{B}$ 已经在多种酯类分子 的加氢中展现出优异的催化性能 ${ }^{[17]}$. 因此, $\mathbf{L}_{5} / \mathrm{Ru}(\mathrm{acac})_{3}$ 的 THF 溶液在反应条件下没有催化活性的原因与 $\mathbf{L}_{\mathbf{1}} /$ $\mathrm{Ru}(\mathrm{acac})_{3}$ 相似(表 2, Entry 8), 可归因于无法原位生成活 性中心. 考虑到反应过程中可能发生甲醇与金属中心作 用生成烷氧基配合物以及分解产生 $\mathrm{CO}$ 与 $\mathrm{Ru}(\mathrm{II})$ 配位 $^{[21]}$, 
导致传统的膦- $\mathrm{Ru}$ 催化剂体系中毒失活. 相比之下, 基 于金属-配体协同作用的化合物 $\mathbf{1}$ (或 2, 3,4) 在 THF 溶剂 中具有较好的催化活性, 无需加入甲醇, 不易造成催化 剂中毒失活, 具有独特的优越性.

围绕化合物 2, Beller 等 ${ }^{[19]}$ 进一步考察了反应条件对 催化性能的影响(表 3). 如 Entry 1 所示, $1 \mathrm{~h}$ 内 2 实现了 DEO 的完全转化, 乙醇酸乙酯和 EG 收率分别为 $21 \%$ 和 $72 \%$. 在不使用 EtONa 条件下, 2 也具有较好的加氢活 性, $1 \mathrm{~h}$ 后乙醇酸乙酯和 $\mathrm{EG}$ 收率分别为 $15 \%$ 和 $85 \%$ (表 3, Entry 2); 4 h 后 EG 收率高于 99\%(表 3, Entry 3). EtONa 等碱金属醇盐在反应过程中, 可以在 $\mathrm{H}_{2}$ 共同作用下将 化合物结构中 $\mathrm{Ru}-\mathrm{Cl}$ 置换为 $\mathrm{RuH}$, 生成催化活性物 种 ${ }^{[22]}$. 这一结果意味着 2 是 1 的催化活性态. 如 Entry 4 所示, 提高底物与催化剂物质的量之比至 $358: 1$, 在 $60{ }^{\circ} \mathrm{C}$ 反应 $1 \mathrm{~h}$ 可以取得 $99 \%$ 的乙醇酸乙酯收率. 进一步 提高底物与催化剂物质的量之比、降低反应温度至室温 或 $\mathrm{H}_{2}$ 压力至 $3.0 \mathrm{MPa}, 2$ 也表现出良好的催化加氢活性 (表 3, Entries 5 7). 最后, 作者也考察了 2 催化 DMO 加氢的反应性能, 如 Entry 8 所示, 反应过程中有较多副 产物生成, EG 收率仅为 $84 \%$.

2014 年, Zhou 课题组 ${ }^{[23]}$ 基于 2,2-联吡啶合成了四齿 PNNN 配体配位的的 Ru(II)化合物 $\mathbf{5}$ (Scheme 2), 并将其

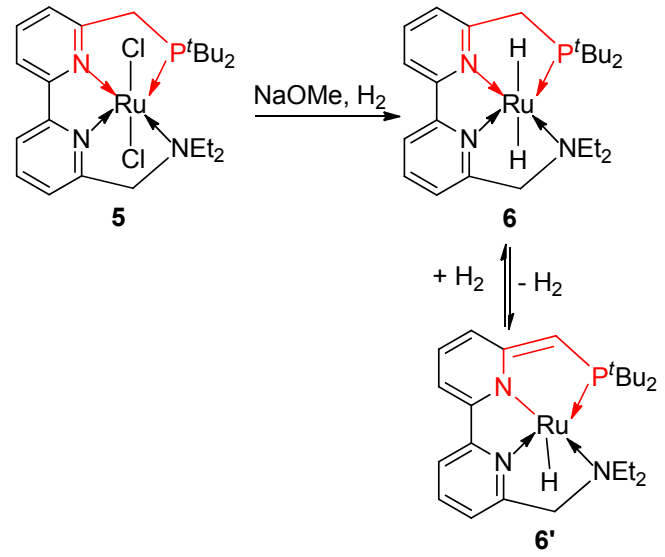

图式 2 化合物 6 和 $6^{\prime}$ 的合成示意图

Scheme 2 Diagram of the synthesis of complexes 6 and $\mathbf{6}^{\prime}$
成功应用于温和条件下多种内酯、脂肪酸酯以及芳香酸 酯的加氢反应研究中. 值得一提的是, 这一化合物能稳 定存在于空气中, 并可以有效催化 DMO 加氢. 在异丙 醇为溶剂、5/ $\mathrm{NaOMe} / \mathrm{DMO}$ 物质的量之为 $1: 100$ : 1000、5.07 $\mathrm{MPa} \mathrm{H}_{2}$ 以及室温等条件下反应 $16 \mathrm{~h}$, 体系中 同时发生加氢反应和酯转移反应，生成了 $99 \%$ 的乙醇酸 异丙醇酯. 提高反应温度至 $100{ }^{\circ} \mathrm{C}, 2 \mathrm{~h}$ 后完全转化为 EG(产率 99\%). 作者利用核磁等表征手段成功证明反应 体系中活性物种 $\mathbf{6}$ 的生成, 并进一步推测了基于配体吡 啶环芳构化/去芳构化过程以及 $\mathrm{RuH}$ 与 “膦臂”亚甲基 氢协同作用的加氢反应机理(Scheme 2).

近年来, 我们课题组 ${ }^{[24]}$ 围绕刚性 $o-\mathrm{PPh}_{2} \mathrm{C}_{6} \mathrm{H}_{4} \mathrm{NH}_{2}$ 配 体 $\left(\mathbf{L}_{5}\right)$, 在 $\mathrm{DMO}$ 均相催化加氢制 $\mathrm{MG} / \mathrm{EG}$ 这一领域也开 展了相关的研究工作. 首先通过 $\left(\mathrm{PPh}_{3}\right)_{3} \mathrm{RuCl}_{2} / n \mathbf{L}_{5}(n=1$, $2,3)$ 体系催化 DMO 加氢的原位活性测试，探究了 $\mathbf{L}_{\mathbf{5}}-\mathrm{Ru}(\mathrm{II})$ 化合物可能形成的结构型式. 从图 1 结果可以 看出, $n$ 为 1 的反应体系在 $48 \mathrm{~h}$ 内就实现了 $\mathrm{DMO}$ 的完 全转化, $n$ 为 2 的反应体系需要约 $96 \mathrm{~h}$; 然而, $n$ 为 3 的反 应体系只能完成约 77\%的 DMO 转化, $48 \mathrm{~h}$ 后基本失活.

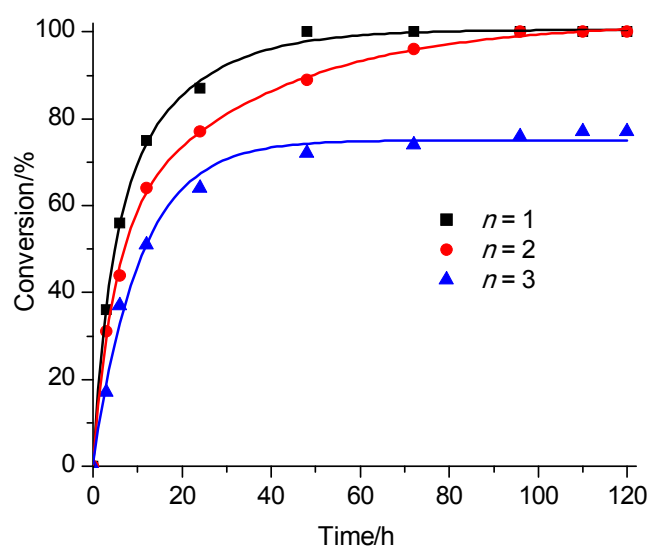

图 $1 \quad\left(\mathrm{PPh}_{3}\right)_{3} \mathrm{RuCl}_{2} / n \mathbf{L}_{\mathbf{5}}$ 催化 $\mathrm{DMO}$ 加氢制 $\mathrm{MG}$ 的反应结果 Figure 1 Catalyzing performance of $\left(\mathrm{PPh}_{3}\right)_{3} \mathrm{RuCl}_{2} / n \mathbf{L}_{5}$ in hydrogenation of DMO into MG

Reaction conditions: $\mathrm{Ru}(0.5 \mathrm{~mol} \%)$, NaOMe (5 mol\%), THF $(10 \mathrm{~mL})$, $p\left(\mathrm{H}_{2}\right)=5.0 \mathrm{MPa}, 100{ }^{\circ} \mathrm{C}$

表 3 不同反应条件下 2 催化 DEO 加氢 ${ }^{a}$

Table 3 Catalytic hydrogenation of DEO with 2 under different reaction conditions

\begin{tabular}{|c|c|c|c|c|c|c|c|c|}
\hline Entry & $n(\mathrm{DEO}) / n(\mathbf{2})$ & Temp. $/{ }^{\circ} \mathrm{C}$ & $p\left(\mathrm{H}_{2}\right) / \mathrm{MPa}$ & Time/h & Conv. $/ \%$ & Yield/\% of ethyl glycolate & Yield $/ \%$ of EG & $\mathrm{TOF}^{d} / \mathrm{h}^{-1}$ \\
\hline $1^{b}$ & 184 & 100 & 6.0 & 1 & 100 & 21 & 72 & 304 \\
\hline 2 & 184 & 100 & 6.0 & 1 & 100 & 15 & 85 & 340 \\
\hline 3 & 184 & 100 & 6.0 & 4 & 100 & 0 & $>99$ & 364 \\
\hline 4 & 358 & 60 & 6.0 & 1 & 99 & 99 & 0 & 354 \\
\hline 5 & 358 & r.t. & 6.0 & 120 & 100 & 7 & 86 & 5 \\
\hline 6 & 358 & 60 & 3.0 & 1 & 50 & 46 & 0 & 165 \\
\hline $7^{b}$ & 6857 & 120 & 6.0 & 16 & 97 & 83 & 3 & 381 \\
\hline $8^{b}$ & $184^{c}$ & 100 & 6.0 & 1 & 100 & 0 & 84 & 309 \\
\hline
\end{tabular}

${ }^{a} \mathrm{THF}$ as solvent; ${ }^{b} \mathrm{NaOEt}(0.2 \mathrm{mmol}) ;{ }^{c} \mathrm{DMO}$ was used; ${ }^{d}$ turnover frequency. 
以上结果说明随着配体用量的增加, 原位反应体系催化 活性逐渐降低. 结合 $\left(\mathrm{PPh}_{3}\right)_{3} \mathrm{RuCl}_{2}$ 不能催化 $\mathrm{DMO}$ 加氢的 实验结果, 我们推测上述原位反应体系中生成了配位有 不同个数 $\mathbf{L}_{\mathbf{5}}$ 配体的 $\mathrm{Ru}(\mathrm{II})$ 化合物.

我们将 $\left(\mathrm{PPh}_{3}\right)_{3} \mathrm{RuCl}_{2}$ 分别与 $1,2,3$ equiv. $\mathbf{L}_{\mathbf{5}}$ 配体反 应，尝试合成出不同结构的 $\mathbf{L}_{5}-\mathrm{Ru}(\mathrm{II})$ 化合物 ${ }^{[24 \mathrm{a}]}$. 在甲 苯溶剂中、 $100{ }^{\circ} \mathrm{C}$ 条件下分别反应 12 和 $48 \mathrm{~h}$ 后, 从 1 和 2 equiv.体系中成功分离得到单/双 $\mathbf{L}_{5}$ 配体配位的化合 物 7 和 8; 3 equiv. 体系在较长反应时间内均仅观察到 8 , 没有新化合物生成. 幸运的是, 以 $\left(\mathrm{PPh}_{3}\right)_{3} \mathrm{RuHCl}$ 作为金 属前驱体与 3 equiv. $\mathbf{L}_{5}$ 配体反应, $24 \mathrm{~h}$ 后制备得到三配 体配位的离子化合物 9. 活性测试结果显示 7 和 8 能有 效催化 DMO 转化为 MG(表 4, Entries 1 \& 2), 9 没有催化 活性(表 4, Entry 3). 9 不能催化 DMO 加氢可以推测主要 是由于金属中心已经配位饱和, 无法活化 $\mathrm{H}_{2}$ 分子产生 RuH. 上述结果说明随着反应的进行, $\left(\mathrm{PPh}_{3}\right)_{3} \mathrm{RuCl}_{2} / 3 \mathbf{L}_{\mathbf{5}}$
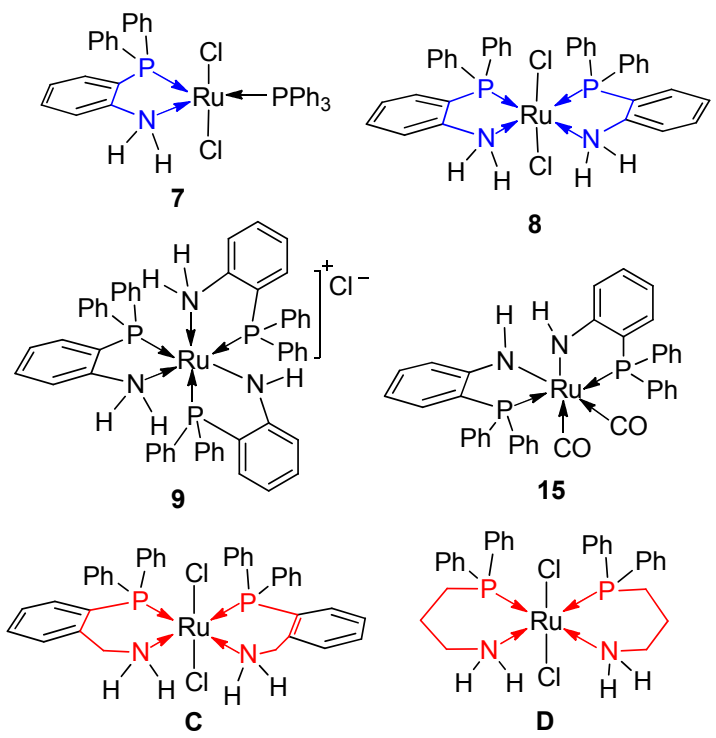

表 4 不同 $\mathrm{Ru}$ 化合物催化 $\mathrm{DMO}$ 加氢制 $\mathrm{MG}^{a}$

Table 4 Catalytic hydrogenation of DMO into MG with different $\mathrm{Ru}$ complexes

\begin{tabular}{cccccc}
\hline Entry & Cat. & $n(\mathrm{NaOMe}) / n(\mathrm{Ru})$ & Time/h & Conv. $\%$ Yield $/ \%$ of $\mathrm{MG}$ \\
\hline 1 & $\mathbf{7}$ & 10 & 1 & 97 & 97 \\
2 & $\mathbf{8}$ & 10 & 1 & 97 & 97 \\
3 & $\mathbf{9}$ & 10 & 1 & 0 & 0 \\
4 & $\mathbf{1 0}$ & 10 & 3 & 100 & 99 \\
5 & $\mathbf{1 2}$ & 0 & 3 & 86 & 86 \\
6 & $\mathbf{1 2}$ & 10 & 3 & 99 & 99 \\
7 & $\mathbf{1 3}$ & 10 & 3 & 0 & 0 \\
8 & $\mathbf{1 4}$ & 10 & 3 & 0 & 0 \\
9 & $\mathbf{1 5}$ & 10 & 3 & 0 & 0 \\
10 & $\mathbf{A}$ & 10 & 4 & 47 & 46 \\
11 & $\mathbf{C}$ & 10 & 4 & 49 & 49 \\
12 & $\mathbf{D}$ & 10 & 4 & 16 & 16 \\
\hline
\end{tabular}

原位反应体系中伴随着单 $\rightarrow$ 双 $\rightarrow$ 三 $\mathbf{L}_{\mathbf{5}}$ 配体配位的化合 物生成, 最终完全转化为 $\mathbf{9}$ (或与 9 具有相同结构特征的 化合物), 致使反应体系失活.

我们进一步以 $\left(\mathrm{PPh}_{3}\right)_{3} \mathrm{RuHCl}(\mathrm{CO})$ 作为金属前驱体 合成了金属中心配位有 1 个羰基分子的化合物 $\mathbf{1 0}$ (Scheme 3). 以 $\mathrm{K}\left[\mathrm{BH}^{s} \mathrm{Bu}_{3}\right]$ 作为氢化试剂, 开展了 $\mathbf{1 0}$ 的 氢化反应并成功分离得到 11. 值得注意的是 11 稳定性 较差, $\mathrm{RuH}$ 易与 $\mathrm{NH}_{2}$ 官能团作用脱去 $\mathrm{H}_{2}$ 分子并二聚生成 12. 有趣的是, 12 在 $\mathrm{H}_{2}$ 气氛以及较低的温度下, 可以通 过 $\mathrm{Ru}-\mathrm{NH}$ 结构基元活化裂解 $\mathrm{H}_{2}$ 分子转化为 $\mathbf{1 1}, \mathrm{Ru}$ 金属 价态不发生变化. 这一过程有效展示了 11 和 12 相互转 化的可逆性. 11 和 12 的单晶结构(图 2) 以及相关的 $\mathrm{D}_{2}$ 实验结果有效验证了这一结论. 作为对比, 我们还合成 了分别与 7 和 8 具有相同分子构型、 $o-\mathrm{PPh}_{2} \mathrm{C}_{6} \mathrm{H}_{4} \mathrm{NMe}_{2}$ 配体配位的 $\left(\mathrm{PPh}_{3}\right)\left(o-\mathrm{PPh}_{2} \mathrm{C}_{6} \mathrm{H}_{4} \mathrm{NMe}_{2}\right) \mathrm{RuCl}_{2}(13)$ 和 $(o-$ $\left.\mathrm{PPh}_{2} \mathrm{C}_{6} \mathrm{H}_{4} \mathrm{NMe}_{2}\right)_{2} \mathrm{RuCl}_{2}$ (14) 以及金属中心配位有 2 个羰 基分子的 15 .

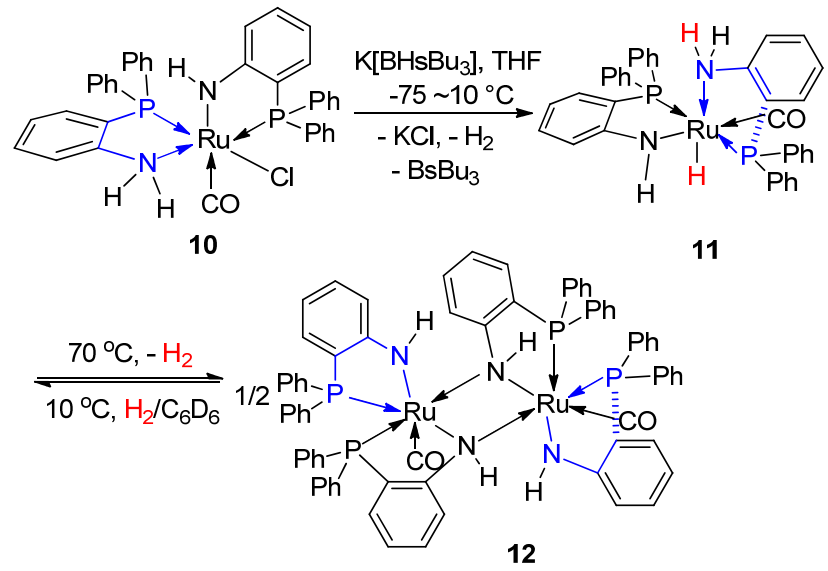

图式 3 化合物 11 和 12 的合成

Scheme 3 Synthesis of complexes $\mathbf{1 1}$ and $\mathbf{1 2}$
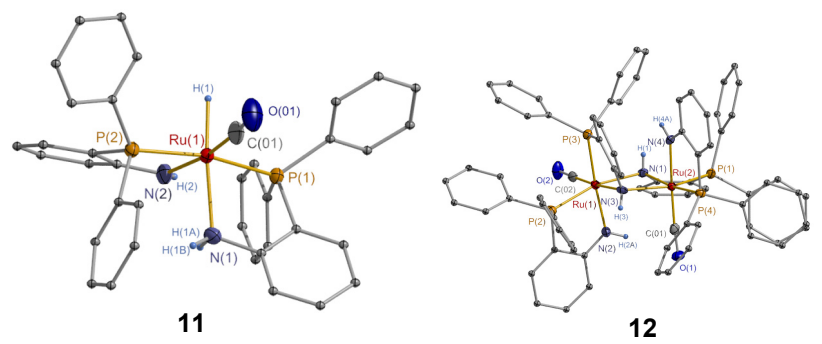

图 2 化合物 11 和 12 的单晶结构

Figure 2 X-ray crystal structures of complexes $\mathbf{1 1}$ and $\mathbf{1 2}$

活性测试结果表明, 10 和 12 可以催化 DMO 部分加 氢制 $\mathrm{MG}$ (表 4, Entries 4, 6,); 此外, 在不使用 $\mathrm{NaOMe}$ 条 件下, 12 也表现出一定的加氢活性(表 4, Entry 5). 考虑 到反应过程中, 10 首先将在 $\mathrm{NaOMe}$ 和 $\mathrm{H}_{2}$ 分子共同作用 下转化成为含有 $\mathrm{RuH}$ 的活性物种 ${ }^{[21 c, 22 b]}$, 同时结合上述 
关于 11 和 12 可逆转化的相关认识, 我们认为 11 是 10 的活性态, 12 为反应中间体. 在相同反应条件下, 不含 $\mathrm{NH}$ 的配合物 13 和 14 均没有催化活性, 这表明 NH 在 反应过程中不可或缺(表 4, Entries 7,8). Noyori、Saudan 和 Ding 等 ${ }^{[17,18 b, 25]}$ 已经报道了类似的实验现象. 化合物 15 与 9 相似, 在相同反应条件下没有催化活性(Entry 9, 表 4). 这一结果有效验证了上文关于 9 不能催化 DMO 加氢的原因所提出的猜测. 综合上述结果可以看出 $\mathrm{RuH}$ 与 $\mathrm{NH}$ 的协同作用在 $\mathbf{L}_{5}-\mathrm{Ru}(\mathrm{II})$ 催化 $\mathrm{DMO}$ 加氢过程中起 着至关重要的作用.

目前, Noyori ${ }^{[14 c]} 、$ Morris ${ }^{[14 b]}$ 以及 Zhou ${ }^{[23]}$ 等均提出了 金属-配体协同作用下 $\mathrm{Ru}(\mathrm{II})$ 催化剂催化苯乙酮、苯甲酸 甲酯等羰基衍生物分子加氢的反应机理. 催化剂通过 $\mathrm{RuH}$ 与配体 $\mathrm{NH}$ (或亚甲基氢)协同作用分别与底物分子 结构中羰基发生亲核和亲电作用, 并进一步将 $\mathrm{RuH}$ 与 $\mathrm{NH}$ 转移至羰基, 实现底物分子的加氢; 形成的催化剂 中间体活化裂解 $\mathrm{H}_{2}$ 分子回到催化活性态. 基于以上研 究, 为了明确草酸酯加氢的反应机理, 我们进行了原位 核磁跟踪监测及同位素实验, 成功检测到 $\mathrm{RuH}$ 的形成 与解离. 同时我们表征了钓氢化合物 11 以及相应中间
体 $\mathbf{1 2}$ 的单晶结构(图 2), 由此推测了 $\mathbf{L}_{5}-\mathrm{Ru}$ (II) 体系催化 DMO 加氢的反应机理 ${ }^{[24 a]}$. 如 Scheme 4 所示, 11 等活性 物种首先通过亲核和亲电协同作用将 $\mathrm{H}^{-} / \mathrm{H}^{+}$等价物转 移到酯基 $\mathrm{C}=\mathrm{O}$ 上, 生成中间体半缩酫，并进一步脱去 一个 $\mathrm{MeOH}$ 分子形成 $\mathrm{MeOC}(\mathrm{O}) \mathrm{CHO}$. 反应生成的催化 剂中间体(如 12 等)活化 $\mathrm{H}_{2}$ 分子重新形成活性态, 再经 催化循环完成 $\mathrm{MeOC}(\mathrm{O}) \mathrm{CHO}$ 醛基加氢生成产物 MG.

我们也测试了柔性膦胺配体构成的 $\mathrm{Ru}(\mathrm{II})$ 化合物 $\left(\mathrm{Ph}_{2} \mathrm{PCH}_{2} \mathrm{CH}_{2} \mathrm{NH}_{2}\right)_{2} \mathrm{RuCl}_{2}(\mathbf{A}) 、 \mathbf{C}$ 以及 $\mathbf{D}$ 催化 $\mathrm{DMO}$ 加氢 的反应活性. A(或 C、D)在 $100{ }^{\circ} \mathrm{C} 、 5.0 \mathrm{MPa} \mathrm{H}_{2}$ 以及 $4 \mathrm{~h}$ 等反应条件下难以有效催化 $\mathrm{DMO}$ 加氢(表 4, Entries $10 \sim 12) . \mathbf{A}$ (和 C、D)结构与 8 相似, 并已被成功用于催 化酮、酯等多种羰基衍生物加氢制醇反应 ${ }^{[17,26]}$. 这些结 果表明了 $\mathbf{L}_{5}-\mathrm{Ru}(\mathrm{II})$ 体系用于 DMO 催化加氢的优越性.

以 8 为例, 我们进一步考察了反应条件对 $\mathrm{L}_{5}-\mathrm{Ru}(\mathrm{II})$ 体系催化加氢性能的影响(表 5). 在较低 $\mathrm{H}_{2}$ 压力、温度 或催化剂用量等反应条件下 8 均可以有效催化 $\mathrm{DMO}$ 部 分加氢制 MG (Entries 1 5). 此外, 提高反应温度和延 长反应时间, 8 也可以催化 DMO 加氢制 EG (Entry 6). 在 $40{ }^{\circ} \mathrm{C} 、 5.0 \mathrm{MPa} \mathrm{H}_{2}$ 以及 $1 \mathrm{~h}$ 等反应条件下，考察了

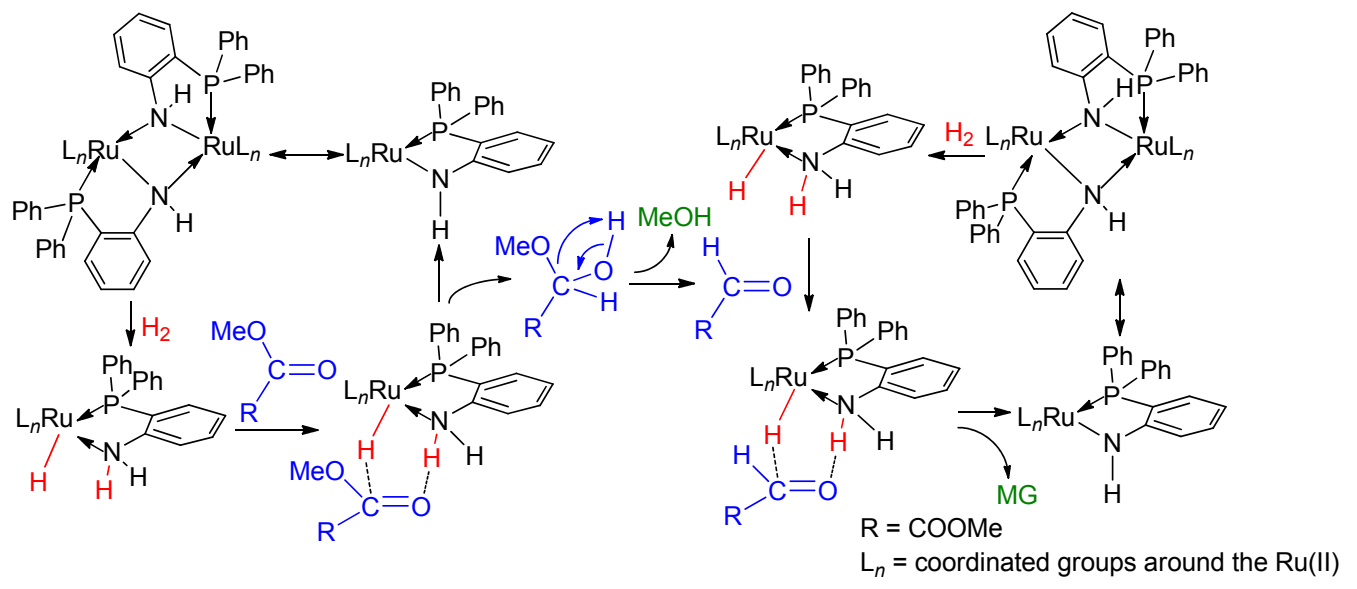

图式 4 推测的催化 DMO 加氢制 MG 的反应机理

Scheme 4 Proposed mechanism for the catalytic hydrogenation of DMO into MG

表 5 不同反应条件下 8 催化 DMO 加氢

Table 5 Catalytic hydrogenation of DMO with 8 in different reaction conditions

\begin{tabular}{|c|c|c|c|c|c|c|c|c|}
\hline Entry & $n(\mathrm{DMO}) / n(\mathbf{8})$ & $n(\mathrm{NaOMe}) / n(\mathbf{8})$ & Temp. $/{ }^{\circ} \mathrm{C}$ & $p\left(\mathrm{H}_{2}\right) / \mathrm{MPa}$ & Time/h & Conv./\% & Yield $/ \%$ of $M G$ & Yield $/ \%$ of EG \\
\hline 1 & 200 & 10 & 100 & 2.0 & 1 & 74 & 74 & 0 \\
\hline 2 & 200 & 10 & 100 & 2.0 & 3 & 98 & 98 & 0 \\
\hline 3 & 200 & 10 & 60 & 5.0 & 1 & 81 & 81 & 0 \\
\hline 4 & 200 & 10 & RT & 5.0 & 24 & 86 & 86 & 0 \\
\hline 5 & 2000 & 10 & 100 & 5.0 & 16 & 98 & 98 & 0 \\
\hline 6 & 100 & 20 & 120 & 5.0 & 36 & 100 & 0 & 97 \\
\hline 7 & 200 & 5 & 40 & 5.0 & 1 & 31 & 31 & 0 \\
\hline 8 & 200 & 10 & 40 & 5.0 & 1 & 35 & 35 & 0 \\
\hline 9 & 200 & 15 & 40 & 5.0 & 1 & 44 & 44 & 0 \\
\hline 10 & 200 & 40 & 40 & 5.0 & 1 & 81 & 81 & 0 \\
\hline 11 & 200 & 80 & 40 & 5.0 & 1 & 65 & 65 & 0 \\
\hline
\end{tabular}


$\mathrm{NaOMe}$ 用量对 8 催化 DMO 加氢活性的影响. 如 Entries 7 11 所示, 催化活性随着 $\mathrm{NaOMe}$ 用量的增加呈现火山 型的变化趋势, 在 $n(\mathrm{NaOMe}) / n(8)$ 等于 40 时最优 $(\mathrm{MG}$ 产 率 $81 \%$, Entry 10). 这些结果表明 $\mathbf{8}$ 与文献报道的催化剂 体系相似，反应过程中助剂碱(如 $\mathrm{NaOMe} 、 t$ - $\mathrm{BuOK}$ 等) 一方面活化催化剂前驱体, 另一方面提升催化加氢活 性 ${ }^{[14 c, 27]}$.

最后, 我们也初步考察了 8 催化其它酯类分子加氢 的反应性能. 结果表明 $\mathbf{8}$ 可以有效催化乳酸甲酯、丙酮 酸甲酯和 $\gamma$-戊内酯等多种酯类分子加氢制醇, 但难以催 化位阻较大的芳香酸酯(如 $\mathrm{MB}$ 等)加氢, 这是一个有趣 的实验现象. 我们注意到 $\mathbf{A}($ 和 $\mathbf{C} 、 \mathbf{D})$ 在催化 $\mathrm{MB}$ 加氢制 苯甲醇(BA)反应中均表现出良好的催化加氢活性. 这些 结果意味着 8 和 $\mathbf{A}$ (或 C、D)在催化 $\mathrm{DMO}$ 及 $\mathrm{MB}$ 加氢反 应中呈现出近乎相反的催化性能. 针对这一现象, 我们 进一步开展了相关的研究工作.

以 8 和 $\mathrm{C}$ 为例进行分析. 如前文所述, 8 (或 $\mathrm{C}$ ) 通过 $\mathrm{RuH}$ 与 $\mathrm{NH}$ 的协同作用实现催化加氢. 对比两者结构可 以看出, 有无 $\mathrm{CH}_{2}$ 链接导致它们 $\mathrm{NH}_{2}$ 官能团局部化学环 境存在一定的差异. 由此可以推测, 这一差异势必影响 它们在催化加氢反应过程中的协同效应, 是造成它们出 现底物选择性的重要原因之一. 根据这一推测, 我们提 出 “构筑一类兼具刚性和柔性结构特征的 $\mathrm{Ru}(\mathrm{II})$ 化合物, 以期该类化合物具有不同结构优势, 表现出良好底物适 用性” 的催化剂设计理念 ${ }^{[24 c]}$.

我们通过分步合成的方法成功制备得到金属中心 同时配位有刚性配体 $\mathbf{L}_{5}$ 和柔性配体 o- $\mathrm{PPh}_{2} \mathrm{C}_{6} \mathrm{H}_{4} \mathrm{CH}_{2} \mathrm{NH}_{2}$ $\left(\mathbf{L}_{6}\right)$ 的新型 $\mathrm{Ru}(\mathrm{II})$ 化合物 $\mathbf{1 6}$. 我们也开展了 $\mathbf{1 6}$ 的 $\mathrm{NaBH}_{4}$ 还原反应, 得到相应的氢化产物 17.16 和 17 的单晶结构 如图 3 所示. 对比两者单晶结构可以看出, 还原前后 $o-\mathrm{PPh}_{2} \mathrm{C}_{6} \mathrm{H}_{4} \mathrm{CH}_{2} \mathrm{NH}_{2}$ 配体中的 $\mathrm{CH}_{2}$ 链接构象发生了翻转, 导致与之相连的 $\mathrm{N}-\mathrm{H}$ 取向发生变化. 这一过程有效地 表明 $o-\mathrm{PPh}_{2} \mathrm{C}_{6} \mathrm{H}_{4} \mathrm{CH}_{2} \mathrm{NH}_{2}-\mathrm{Ru}$ 结构单元较为柔软的特点. 我们进一步通过分步合成的方法制备了与 16 具有相同 骨架结构、 $\mathbf{L}_{5}$ 或 $\mathbf{L}_{6}$ 配体 $\mathrm{NH}$ 分别被甲基化的化合物( $o-$ $\left.\mathrm{PPh}_{2} \mathrm{C}_{6} \mathrm{H}_{4} \mathrm{CH}_{2} \mathrm{NH}_{2}\right)\left(o-\mathrm{PPh}_{2} \mathrm{C}_{6} \mathrm{H}_{4} \mathrm{NMe}_{2}\right) \mathrm{RuCl}_{2}(\mathbf{1 8})$ 和 $(o-\mathrm{P}-$

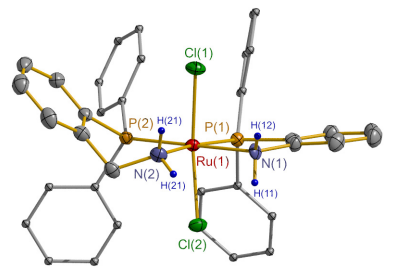

16

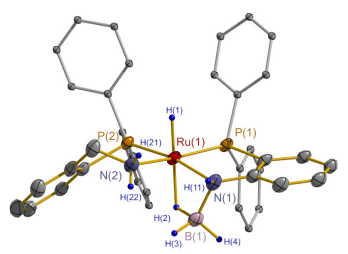

17
图 3 化合物 16 和 17 的单晶结构

Figure 3 X-ray crystal structures of complexes 16 and 17
$\left.\mathrm{Ph}_{2} \mathrm{C}_{6} \mathrm{H}_{4} \mathrm{CH}_{2} \mathrm{NMe}_{2}\right)\left(o-\mathrm{PPh}_{2} \mathrm{C}_{6} \mathrm{H}_{4} \mathrm{NH}_{2}\right) \mathrm{RuCl}_{2}$ (19).

分别考察了化合物 16 19 催化 $\mathrm{DMO}$ 和 $\mathrm{MB}$ 加氢 的反应性能. 如表 6 所示, 相比于 $\mathbf{8}$ (或 $\mathrm{C}$ )仅能有效催化 $\mathrm{DMO}$ (或 $\mathrm{MB}$ )加氢, 16 在 $\mathrm{DMO}$ 和 $\mathrm{MB}$ 加氢反应中均表 现出良好的催化活性(Entries 1，2). 进一步活性测试结 果显示 16 可以有效催化多种不同结构内酯、脂肪酸酯 和芳香酸酯加氢制相应的醇; 此外, 在酰胺以及碳酸酯 的加氢反应中也表现出一定的催化活性. 这些结果表明 16 兼具 8 和 $C$ 分别在 $D M O$ 和 MB 加氢中的优势, 具有 良好的底物适用性, 有力验证了我们设计催化剂理念的 正确性和有效性.

表 6 催化 $\mathrm{DMO}$ 加氢制 $\mathrm{MG}$ (和/或 $\mathrm{EG}$ )和 $\mathrm{MB}$ 加氢制 $\mathrm{BA}^{a}$ Table 6 Catalytic hydrogenation of DMO into MG (and/or EG) and that of MB into BA

\begin{tabular}{cccclc}
\hline \multirow{2}{*}{ Entry } & \multirow{2}{*}{ Cat. } & \multirow{2}{*}{ Substrate } & Conv./\% & \multicolumn{2}{c}{ Yield/\% } \\
\cline { 5 - 6 } & & & & MG or BA & EG \\
\hline 2 & $\mathbf{1 6}$ & DMO & 100 & $93(\mathrm{MG})$ & 6 \\
3 & & MB & 95 & $93(\mathrm{BA})$ & \\
4 & $\mathbf{1 7}$ & DMO & 100 & $91(\mathrm{MG})$ & 7 \\
$5^{b}$ & & MB & 97 & $96(\mathrm{BA})$ & \\
$6^{b}$ & $\mathbf{1 7}$ & DMO & 100 & $100(\mathrm{MG})$ & 0 \\
7 & & MB & 7 & $5(\mathrm{BA})$ & \\
8 & $\mathbf{1 8}$ & DMO & 0 & $0(\mathrm{MG})$ & 0 \\
9 & & MB & 90 & $88(\mathrm{BA})$ & \\
10 & $\mathbf{1 9}$ & DMO & 80 & $79(\mathrm{MG})$ & 0 \\
11 & & MB & 3 & $1(\mathrm{BA})$ & \\
12 & $\mathbf{2 0}$ & DMO & 100 & $86(\mathrm{MG})$ & 13 \\
13 & \multirow{21}{*}{} & MB & 100 & $100(\mathrm{BA})$ & \\
14 & & DMO & 100 & $100(\mathrm{MG})$ & 0 \\
\hline
\end{tabular}

${ }^{a}$ Reaction conditions: $0.5 \mathrm{~mol} \% \mathrm{Ru}, 5 \mathrm{~mol} \%$ (for DMO) or $10 \mathrm{~mol} \%$ (for $\mathrm{MB}$ ) NaOMe, THF $(10 \mathrm{~mL}), p\left(\mathrm{H}_{2}\right)=5.0 \mathrm{MPa}, 100{ }^{\circ} \mathrm{C}, 4 \mathrm{~h} ;{ }^{b}$ No NaOMe was used.

如表 6 所示, 化合物 17 催化活性与 $\mathbf{1 6}$ 相当(Entries 3, 4). 在不使用 $\mathrm{NaOMe}$ 条件下, 17 也可以有效催化 $\mathrm{DMO}$ 加氢, 但在 MB 加氢反应中活性较低(Entries 5, 6). 这一结果意味着配合物 $\mathbf{1 6}$ 与 8 等相似, 需要一定量 的碱提升其催化加氢活性. 与 16 形成鲜明对比, 18 (或 19)在反应条件下仅能催化 $\mathrm{MB}$ (或 DMO)加氢(Entries $7 \sim 10)$. 这些结果表明结构基元 $\mathbf{L}_{5}-\mathrm{Ru}$ 和 $\mathbf{L}_{6}-\mathrm{Ru}$ 保留各 自的性质特点, 16 兼具刚性结构和柔性结构分别参与协 同作用的优势. 这一优势是它能有效催化 $\mathrm{DMO} 、 \mathrm{MB}$ 等 不同底物分子加氢的直接原因.

进一步采用柔性配体 $\mathrm{Ph}_{2} \mathrm{PCH}_{2} \mathrm{CH}_{2} \mathrm{NH}_{2}$ 和 $\mathrm{Ph}_{2} \mathrm{P}$ $\left(\mathrm{CH}_{2}\right)_{3} \mathrm{NH}_{2}$, 分别与 $\mathbf{L}_{5}$ 组合制备了新型 $\mathrm{Ru}(\mathrm{II})$ 化合物 $\mathbf{2 0}$ 和 21. 如表 6 所示, 20 (或 21) 在 DMO 和 MB 加氢反应 中均表现出较好的催化活性(Entries 11 14). 这些结果 意味着新型 $\mathrm{Ru}(\mathrm{II})$ 化合物的设计、合成及应用具有一定 
的普适性.
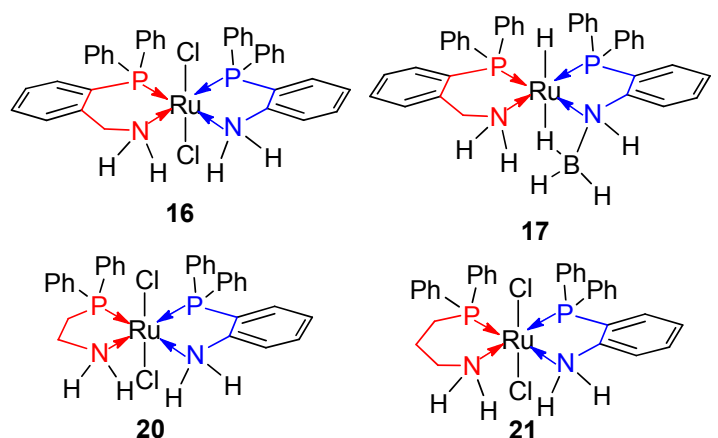

17

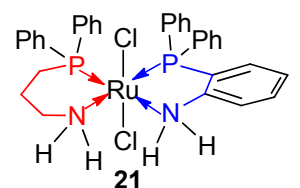

如前文所述，具有金属-配体协同作用 $\mathrm{Ru}(\mathrm{II})$ 化合 物在参与反应过程中多需要大量的助剂碱提升催化加 氢活性 ${ }^{[14 \mathrm{c}, 27 \mathrm{a}]}$. 然而, 过量助剂的使用会带来多方面不 利的影响, 如增加反应成本和废弃物排放等. 探索开发 少/无助剂型 $\mathrm{Ru}(\mathrm{II})$ 催化剂从学术研究和工业应用的角 度均具有重要的意义. 目前, 研究者针对碱提升催化加 氢活性的原因已经开展了多方面的研究工作. 例如, Bergens 课题组 ${ }^{[28]}$ 报道指出反应过程中碱金属阳离子可 以部分取代配体 $\mathrm{NH}$ 形成 $\mathrm{N}^{\delta-}-\mathrm{M}^{\delta+}$ 结构单元(Scheme 5). 作者认为 $\mathrm{N}^{\delta-}-\mathrm{M}^{\delta+}$ 结构单元的生成增加了钉金属中心的 电子云密度和 $\mathrm{RuH}$ 亲核性, 增强了 $\mathrm{RuH}$ 活化 $\mathrm{C}=\mathrm{O}$ 的 能力, 进而实现催化活性的提升. 我们注意到有关催化 加氢反应机理的研究已经明确提出 $\mathrm{Ru}(\mathrm{II})$ 催化剂活性与 $\mathrm{RuH}$ 亲核性间的线性关系 ${ }^{[14 c, 29]}$. 此外, Kayaki 等 ${ }^{[30]}$ 报道 的化合物 1 结构中 $\mathrm{CO}$ 被富电子 $N$-杂环卡宾替代所得到 新化合物具有更高催化活性的实验结果进一步验证了 上述观点.

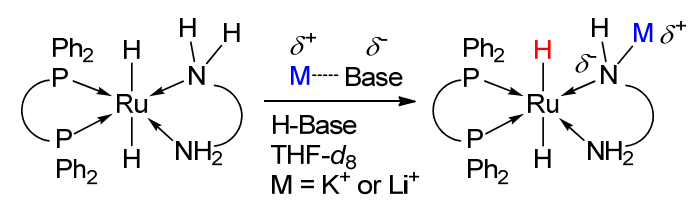

图式 5 碱金属阳离子取代胺基氢

Scheme 5 Substitution of amino hydrogen by an alkali metal cation

基于上述研究进展, 我们提出在位阻允许的范围内 将传统 $\mathrm{NH}_{2}-\mathrm{Ru}(\mathrm{II})$ 催化剂结构中伯胺官能团替代为供电 性烷基取代的仲胺官能团, 希望通过 $\mathrm{N}-\mathrm{R}$ 结构基元在 一定程度上模拟原位形成 $\mathrm{N}^{\delta-}-\mathrm{M}^{\delta+}$ 的效果, 在提升催化 加氢活性的同时降低取得最优催化活性所需的助剂用 量 ${ }^{[24 d]}$. 根据这一设想, 我们制备了两类仲胺基配体配 位的 $\mathrm{Ru}(\mathrm{II})$ 化合物. 一方面, 采用单烷基取代的配体 $o-\mathrm{PPh}_{2} \mathrm{C}_{6} \mathrm{H}_{4} \mathrm{NHR}\left(\mathrm{Me}, \mathrm{Et}, \mathrm{CH}_{2} \mathrm{Ph}\right)$, 成功制备出化合物 22 24. 另一方面, 采用乙二胺以及 $N, N^{\prime}$-二烷基取代的 乙二胺配体 $\left(\mathrm{CH}_{2} \mathrm{NHR}\right)_{2}\left(\mathrm{R}=\mathrm{H}, \mathrm{Me}, \mathrm{Et},{ }^{i} \mathrm{Pr}\right)$, 制备得到化
合物 25 28. 值得注意的是，二胺基配体配位的钉催化 剂虽然已经成功应用于催化醛、酮加氢 ${ }^{[14 a, 31]}$, 但用于酯 类分子催化加氢还鲜有报道.

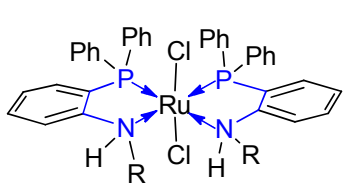

22: $\mathrm{R}=\mathrm{Me}$

23: $\mathrm{R}=\mathrm{Et}$

24: $\mathrm{R}=\mathrm{CH}_{2} \mathrm{Ph}$

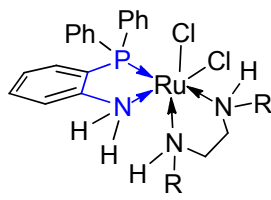

25: $\mathrm{R}=\mathrm{H}$

26: $R=M e$

27: $\mathrm{R}=\mathrm{Et}$

28: $\mathrm{R}={ }^{i} \mathrm{Pr}$
在 $0.05 \mathrm{~mol} \% \mathrm{Ru} 、 0.5 \mathrm{~mol} \% \mathrm{NaOMe} 、 5.0 \mathrm{MPa} \mathrm{H}_{2}$ 以及 $100{ }^{\circ} \mathrm{C}$ 等反应条件下考察了 $8 、 \mathbf{2 2} \sim \mathbf{2 8}$ 催化 $\mathrm{DMO}$ 加氢的反应性能. 如表 7 所示, 22 24 的催化活性相比 于 8 均有了不同程度的提升, 其中以 22 最优(表 7, Entries 2 4). 计算可得 22 相应的 TOF 值为 $1520 \mathrm{~h}^{-1}$ (表 7, Entry 2)，远高于 8(表 7, Entry 1). 二胺配体构成的化 合物 25 28 呈现出不同的催化活性. 乙二胺配体配位 的化合物 25 在反应条件下没有催化活性(表 7, Entry 5). 相比之下, $N, N^{\prime}$-二甲基乙二胺和 $N, N^{\prime}$-二乙基乙二胺配 位的化合物 26 和 27 催化活性截然不同, 分别取得了 36\% 和 98\%的 MG 收率(表 7, Entries 6, 7). 此外, 在 27 参与催化的反应体系中还检测到少量 $\mathrm{EG}$ 生成(产率 $1 \%$, 表 7, Entry 7). 事实上, 在 $0.5 \mathrm{~h}$ 内 27 就已经基本实现了 DMO 的完全转化, TOF 值达到 $3920 \mathrm{~h}^{-1}$ (表 7, Entry 8). 据我们所知这是目前催化 DMO 部分加氢制 MG 所取得 的最好实验结果. 对比 Entries 5 7 结果可以看出, 在 一定程度上催化活性随着胺配位基电子云密度的增加 而增加. 这些结果进一步验证了 Bergens 课题组 ${ }^{[28]}$ 所提 出的观点. 由于异丙基位阻较大, 28 催化活性较低, 仅 取得 $6 \%$ 的 DMO 转化率(表 7, Entry 9).

表 7 不同 $\mathrm{Ru}$ 化合物催化 $\mathrm{DMO}$ 加氢 ${ }^{a}$

Table 7 Catalytic hydrogenation of DMO with different $\mathrm{Ru}$ complexes

\begin{tabular}{cccccc}
\hline \multirow{2}{*}{ Entry } & \multirow{2}{*}{ Cat. } & \multirow{2}{*}{ Conv./\% } & \multicolumn{2}{c}{ Yield/\% } & \multirow{2}{*}{ TOF $/ \mathrm{h}^{-1}$} \\
\cline { 4 - 5 } & & & MG & EG & \\
\hline 1 & $\mathbf{8}$ & 8 & 8 & 0 & 160 \\
2 & $\mathbf{2 2}$ & 77 & 76 & 0 & 1520 \\
3 & $\mathbf{2 3}$ & 27 & 27 & 0 & 540 \\
4 & $\mathbf{2 4}$ & 24 & 24 & 0 & 480 \\
5 & $\mathbf{2 5}$ & 0 & 0 & 0 & - \\
6 & $\mathbf{2 6}$ & 36 & 36 & 0 & 720 \\
7 & $\mathbf{2 7}$ & 100 & 98 & 1 & 2000 \\
$8^{b}$ & $\mathbf{2 7}$ & 99 & 98 & 0 & 3920 \\
9 & $\mathbf{2 8}$ & 6 & 6 & 0 & 120 \\
\hline
\end{tabular}

${ }^{a}$ Reaction conditions: $0.05 \mathrm{~mol} \% \mathrm{Ru}, 0.5 \mathrm{~mol} \% \mathrm{NaOMe}$, THF $(10 \mathrm{~mL})$, $p\left(\mathrm{H}_{2}\right)=5.0 \mathrm{MPa}, 100{ }^{\circ} \mathrm{C}, 1 \mathrm{~h} ;{ }^{b} 0.5 \mathrm{~h}$.

我们也进一步考察了 8 以及 $22 \sim 28$ 催化苯甲醛(或 苯乙酮)加氢制 $\mathrm{BA}$ (或 1-苯乙醇)的反应活性. 有趣的是, 
催化活性均呈现在 DMO 催化加氢反应中相似的变化趋 势. 综合上述结果可以看出, 在位阻效应允许的范围内 通过将 $\mathrm{NH}_{2}-\mathrm{Ru}(\mathrm{II})$ 催化剂结构中伯胺官能团替代为富电 性仲胺官能团来提升催化活性这一设想是可行的.

最后, 围绕 27 考察了反应条件对催化 DMO 加氢性 能的影响. 如表 8 所示, 催化活性随着 $\mathrm{NaOMe}$ 用量的增 加呈现出先增加后降低的火山型变化趋势, 并在 $n(\mathrm{NaOMe}) / n$ (27)等于 10 时取得峰值(表 8, Entries 1 4). 虽然这一变化趋势与 8 等相似, 但相比之下 27 取得最优 催化活性所需助剂用量显著降低. 这一结果意味着针对 $\mathrm{NH}_{2}-\mathrm{Ru}(\mathrm{II})$ 催化剂可以通过将伯胺官能团替代为供电性 烷基取代的仲胺官能团这一方式来降低催化剂所需助 剂的用量 ${ }^{[27 \mathrm{a}-27 \mathrm{~b}]}$. 在较低温度和 $\mathrm{H}_{2}$ 压力条件下, 27 也可 以有效催化 $\mathrm{DMO}$ 加氢制 $\mathrm{MG}$ (表 8 , Entries 5 8). 值得 注意的是，在室温条件下反应 $20 \mathrm{~h}$ 可以取得 $95 \%$ 的 MG 收率, 相应的 TOF 值为 $24 \mathrm{~h}^{-1}$ (表 8, Entry 6). 这一数值 远高于 $\mathbf{2}$ 在相似反应条件下催化草酸二乙酯加氢的实验 结果(Entry 5, 表 3). 我们也考察了 27 催化 DMO 加氢制 EG 的反应性能, 结果显示催化活性相比于 $\mathbf{8}$ 也有显著 的提升(表 8, Entries 9, $10 \mathrm{vs}$ 表 5, Entry 6).

\section{2 结论与展望}

开发可高效催化草酸酯加氢制乙二醇的催化剂是 “煤制乙二醇”工艺路线的研究热点和难点. 在过去的近 10 年里, 基于金属-配体协同作用的 $\mathrm{Ru}(\mathrm{II})$ 催化剂用于 草酸酯均相催化加氢的研究取得了一定的进展, 也发展
制备出一系列结构各异的 $\mathrm{Ru}(\mathrm{II})$ 催化剂. 研究结果表明, 配体结构对金属-配体间协同作用的发挥乃至催化剂催 化加氢性能有显著影响. 在尽可能降低位阻效应的前提 下配体与金属中心配位所形成的结构单元具有一定刚 性为佳; 胺配位基具有高的电子云密度，与金属中心配 位后能有效提升 $\mathrm{RuH}$ 亲核性. 在反应过程中, $\mathrm{RuH}$ 与配 体 $\mathrm{NH}$ (或亚甲基氢)两者均不可或缺; 配体提供 $\mathrm{NH}$ (或 亚甲基氢() $\mathrm{RuH}$ 产生协同效应，并分别通过亲电和亲 核作用将 $\mathrm{H}^{+} / \mathrm{H}^{-}$等价物转移至底物分子羰基上，实现催 化加氢; 生成的催化剂中间体通过 Ru-NH 等结构基元 活化裂解 $\mathrm{H}_{2}$ 分子重新形成活性态. 具有金属-配体协同 作用 $\mathrm{Ru}(\mathrm{II})$ 催化剂相比于传统的膦- $\mathrm{Ru}$ 催化剂体系具有 更优异的催化加氢活性和稳定性，同时所需反应条件较 为温和.

针对现有均相配合物催化剂难以分离以及循环使 用等问题，接下来可以通过配体修饰，以物理或化学作 用的形式将催化剂针定在金属一有机框架或有机聚合物 等材料上，探索开展金属-配体协同作用 $\mathrm{Ru}(\mathrm{II})$ 催化剂 固载化的研究. 均相催化剂固载化保留了金属配合物活 性位点的结构和性质, 实现了均相催化剂反应条件温 和、催化效率高和非均相催化剂使用寿命长、易于分离 回收再利用等优势的有机结合; 此外，也有利于从分子 层面设计新催化剂. 鉴于研究人员已经成功实现多种类 型均相催化剂的固载化，相信在不久的将来固载型金 属-配体协同作用 $\mathrm{Ru}(\mathrm{II})$ 催化剂的合成及应用研究有望 取得实质性的进展.

表 8 不同反应条件下 27 催化 DMO 加氢

Table 8 Catalytic hydrogenation of DMO with 27 under different reaction conditions

\begin{tabular}{|c|c|c|c|c|c|c|c|c|c|}
\hline \multirow{2}{*}{ Entry } & \multirow{2}{*}{$n(\mathrm{DMO}) / n(\mathbf{2 7})$} & \multirow{2}{*}{$n(\mathrm{NaOMe}) / n(\mathbf{2 7})$} & \multirow{2}{*}{ Temp. $/{ }^{\circ} \mathrm{C}$} & \multirow{2}{*}{$p\left(\mathrm{H}_{2}\right) / \mathrm{MPa}$} & \multirow{2}{*}{ Time/h } & \multirow{2}{*}{ Conv. $/ \%$} & \multicolumn{2}{|c|}{ Yield $/ \%$} & \multirow{2}{*}{$\mathrm{TOF} / \mathrm{h}^{-}$} \\
\hline & & & & & & & MG & EG & \\
\hline 1 & 2000 & 5 & 100 & 5.0 & 1 & 87 & 86 & 0 & 1720 \\
\hline 3 & 2000 & 10 & 100 & 5.0 & 1 & 100 & 98 & 1 & 2000 \\
\hline 3 & 2000 & 15 & 100 & 5.0 & 1 & 87 & 86 & 0 & 1720 \\
\hline 4 & 2000 & 20 & 100 & 5.0 & 1 & 68 & 67 & 0 & 1340 \\
\hline 5 & 500 & 10 & 100 & 1.0 & 1.5 & 100 & 99 & 0 & 330 \\
\hline 6 & 500 & 10 & r.t. & 5.0 & 20 & 96 & 95 & 0 & 24 \\
\hline 7 & 500 & 10 & 40 & 5.0 & 2 & 100 & 99 & 0 & 247 \\
\hline 8 & 500 & 10 & 40 & 1.0 & 16 & 95 & 94 & 0 & 29 \\
\hline 9 & 500 & 10 & 120 & 5.0 & 4 & 100 & 0 & 97 & 242 \\
\hline 10 & 200 & 10 & 100 & 5.0 & 4 & 100 & 0 & 99 & 99 \\
\hline
\end{tabular}

\section{References}

[1] Chen, L.; Guo, P.; Qiao, M.; Yan, S.; Li, H.; Wei, S.; Xu, H.; Fan, K. J. Catal. 2008, 257, 172.

[2] Yin, G. Sino-Global Energy 2012, 17, 62 (in Chinese). (尹国海，中外能源, 2012, 17, 62.)

[3] (a) Liu, Z. Chem. Ind. Eng. Prog. 2013, 32, 1214 (in Chinese). (刘宗语，化工进展, 2013, 32, 1214.)

(b) Chen, W.; Sun, J.; Zhang, J.; Zhang, S.; Hua, W. Chem. Ind. Eng. Prog. 2014, 33, 1740 (in Chinese).
(成卫国，孙剑，张军平，张锁江，华炜，化工进展，2014，33， 1740.)

[4] (a) Zheng, J.; Lin, H.; Wang, Y.; Zheng, X.; Duan, X.; Yuan, Y. J. Catal. 2013, 297, 110.

(b) He, Z.; Lin, H.; He, P.; Yuan, Y. J. Catal. 2011, 277, 54.

(c) Xu, C.; Chen, G.; Zhao, Y.; Liu, P.; Duan, X.; Gu, L.; Fu, G.; Yuan, Y.; Zheng, N. Nat. Commun. 2018, 9, 3367.

[5] Grey, R. A.; Pez, G. P.; Wallo, A. J. Am. Chem. Soc. 1981, 103, 7536.

[6] (a) Turek, T.; Trimm, D. L.; Cant, N. W. Catal. Rev.: Sci. Eng. 
1994, 36, 645 .

(b) Pouilloux, Y.; Autin, F.; Barrault, J. Catal. Today 2000, 63, 87.

(c) Wang, H.; Zhang, T.; Zhou, X. J. Phys.: Condens. Matter 2019, 31,473001

[7] Grey, R. A.; Pez, G. P.; Wallo, A.; Corsi, J. J. Chem. Soc., Chem. Commun. 1980, 783.

[8] Matteoli, U.; Blanchi, M.; Menchi, G.; Prediani, P.; Piacenti, F. J. Mol. Catal. 1984, 22, 353.

[9] (a) Matteoli, U.; Bianchi, M.; Menchi, G.; Frediani, P.; Piacenti, F. J. Mol. Catal. 1985, 29, 269.

(b) Matteoli, U.; Menchi, G.; Bianchi, M.; Piacenti, F. J. Organomet. Chem. 1986, 299, 233.

[10] Teunissen, H. T.; J. Elsevier, C. Chem. Commun. 1997, 667.

[11] (a) Teunissen, H. T. Chem. Commun. 1998, 1367.

(b) van Engelen, M. C.; Teunissen, H. T.; de Vries, J. G.; Elsevier, C. J. J. Mol. Catal. A: Chem. 2003, 206, 185.

[12] Boardman, B.; Hanton, M. J.; Rensburg, H. V.; Tooze, R. P. Chem. Commun. 2006, 2289.

[13] Ohkuma, T.; Ooka, H.; Ikariya, T.; Noyori, R. J. Am. Chem. Soc. 1995, 117,10417

[14] (a) Doucet, H.; Ohkuma, T.; Murata, K.; Yokozawa, T.; Kozawa, M.; Katayama, E.; England, A. F.; Ikariya, T.; Noyori, R. Angew. Chem., Int. Ed. 1998, 37, 1703.

(b) Abdur-Rashid, K.; Clapham, S. E.; Hadzovic, A.; Harvey, J. N.; Lough, A. J.; Morris, R. H. J. Am. Chem. Soc. 2002, 124, 15104.

(c) Sandoval, C. A.; Ohkuma, T.; Muñiz, K.; Noyori, R. J. Am. Chem. Soc. 2003, 125, 13490.

(d) Ikariya, T.; Murata, K.; Noyori, R. Org. Biomol. Chem. 2006, 4, 393

(e) Dub, P. A.; Gordon, J. C. Nat. Rev. Chem. 2018, 2, 396.

[15] (a) Clapham, S. E.; Hadzovic, A.; Morris, R. H. Coord. Chem. Rev. 2004, 248, 2201.

(b) vom Stein, T.; Meuresch, M.; Limper, D.; Schmitz, M.; Hölscher, M.; Coetzee, J.; Cole-Hamilton, D. J.; Klankermayer, J.; Leitner, W. J. Am. Chem. Soc. 2014, 136, 13217.

(c) Liu, Y.; Yue, X.; Luo, C.; Zhang, L.; Lei, M. Energy Environ. Mater. 2019, 2, 292.

[16] (a) Dub, P. A.; Ikariya, T. ACS Catal. 2012, 2, 1718.

(b) Zhao, B.; Han, Z.; Ding, K. Angew. Chem., Int. Ed. 2013, 52, 4744 .

(c) Werkmeister, S.; Junge, K.; Beller, M. Org. Process Res. Dev. 2014, 18, 289.

(d) Pritchard, J.; Filonenko, G. A.; van Putten, R.; Hensen, E. J. M.; Pidko, E. A. Chem. Soc. Rev. 2015, 44, 3808.

(e) Zhou, Y.; Khan, R.; Fan, B.; Xu, L. Synthesis 2019, 51, 2491.

(f) Dub, P. A.; Batrice, R. J.; Gordon, J. C.; Scott, B. L.; Minko, Y.; Schmidt, J. G.; Williams, R. F. Org. Process Res. Dev. 2020, 24, 415.

[17] Saudan, L. A.; Saudan, C. M.; Debieux, C.; Wyss, P. Angew. Chem., Int. Ed. 2007, 46, 7473.

[18] (a) Kuriyama, W.; Matsumoto, T.; Ogata, O.; Ino, Y.; Aoki, K.; Tanaka, S.; Ishida, K.; Kobayashi, T.; Sayo, N.; Saito, T. Org. Process Res. Dev. 2012, 16, 166.

(b) Han, Z.; Rong, L.; Wu, J.; Zhang, L.; Wang, Z.; Ding, K. Angew. Chem., Int. Ed. 2012, 51, 13041.
[19] Ziebart, C.; Jackstell, R.; Beller, M. ChemCatChem 2013, 5.

[20] Spasyuk, D.; Smith, S.; Gusev, D. G. Angew. Chem., Int. Ed. 2012, $51,2772$.

[21] (a) Van der Sluys, L. S.; Kubas, G. J.; Caulton, K. G. Organometallics 1991, 10, 1033.

(b) Chen, Y. Z.; Chan, W. C.; Lau, C. P.; Chu, H. S.; Lee, H. L.; Jia, G. Organometallics 1997, 16, 1241

(c) Hamilton, R. J.; Bergens, S. H. J. Am. Chem. Soc. 2006, 128 , 13700

(d) Shen, Y.; Zhan, Y.; Li, S.; Ning, F.; Du, Y.; Huang, Y.; He, T.; Zhou, X. ChemSusChem 2018, 11, 864.

(e) Fang, X.; Duan, N.; Zhang, M.; Zhang, C.; Liu, R.; Zhu, H. P. Chin. J. Org. Chem. 2019, 39, 1450 (in Chinese).

(方霄龙, 段宁，章敏，张春燕，刘睿，朱红平，有机化学，2019, 39,1450 .)

[22] (a) Abdur-Rashid, K.; Faatz, M.; Lough, A. J.; Morris, R. H. J. Am. Chem. Soc. 2001, 123, 7473.

(b) Hartmann, R.; Chen, P. Angew. Chem., Int. Ed. 2001, 40, 3581.

[23] Li, W.; Xie, J. H.; Yuan, M. L.; Zhou, Q. L. Green Chem. 2014, 16, 4081.

[24] (a) Fang, X.; Zhang, C.; Chen, J.; Zhu, H.; Yuan, Y. RSC Adv. 2016, 6,45512 .

(b) Zhang, Y. W.; Chen, Y. L.; Fang, X. L.; Yuan, Y. Z.; Zhu, H. P. Chin. J. Org. Chem. 2017, 37, 2275 (in Chinese)

(张亦伟, 陈艺林, 方霄龙, 袁友珠, 朱红平, 有机化学, 2017, 37, 2275.)

(c) Fang, X.; Sun, M.; Zheng, J.; Li, B.; Ye, L.; Wang, X.; Cao, Z.; Zhu, H.; Yuan, Y. Sci. Rep. 2017, 7, 3961.

(d) Fang, X.; Li, B.; Zheng, J.; Wang, X.; Zhu, H.; Yuan, Y. Dalton Trans. 2019, 48, 2290.

(e) Fang, X.; Zhang, M.; Duan, N.; Wang, X.; Zhu, H. P. Chin. J. Org. Chem. 2020, 40, 226 (in Chinese).

(方霄龙, 章敏, 段宁, 汪新, 朱红平, 有机化学, 2020, 40, 226.)

[25] Noyori, R.; Ohkuma, T. Angew. Chem., Int. Ed. 2001, 40, 40.

[26] (a) Abdur-Rashid, K.; Guo, R.; Lough, A. J.; Morris, R. H.; Song, D. Adv. Synth. Catal. 2005, 347, 571

(b) Jia, W.; Chen, X.; Guo, R.; Sui-Seng, C.; Amoroso, D.; Lough, A. J.; Abdur-Rashid, K. Dalton Trans. 2009, 39, 8301.

[27] (a) Drake, J. L.; Manna, C. M.; Byers, J. A. Organometallics 2013 , 32,6891 .

(b) Spasyuk, D.; Smith, S.; Gusev, D. G. Angew. Chem., Int. Ed. 2013, 52, 2538.

(c) Moore, C. M.; Bark, B.; Szymczak, N. K. ACS Catal. 2016, 6, 1981.

[28] John, J. M.; Takebayashi, S.; Dabral, N.; Miskolzie, M.; Bergens, S. H. J. Am. Chem. Soc. 2013, 135, 8578.

[29] Dub, P. A.; Henson, N. J.; Martin, R. L.; Gordon, J. C. J. Am. Chem. Soc. 2014, 136, 3505.

[30] Ogata, O.; Nakayama, Y.; Nara, H.; Fujiwhara, M.; Kayaki, Y. Org. Lett. 2016, 18, 3894.

[31] (a) Ohkuma, T.; Koizumi, M.; Muñiz, K.; Hilt, G.; Kabuto, C.; Noyori, R. J. Am. Chem. Soc. 2002, 124, 6508.

(b) Guo, R.; Chen, X.; Elpelt, C.; Song, D.; Morris, R. H. Org. Lett. 2005, 7, 1757. 